OPEN ACCESS

Edited by:

Craig N. Jenne,

University of Calgary, Canada

Reviewed by:

Mia Phillipson,

Uppsala University, Sweden

Victor Naumenko,

National University of Science and Technology MISiS, Russia

*Correspondence:

Sven Brandau

sven.brandau@uk-essen.de

tPresent Address:

Anika Grüneboom,

Department of Internal Medicine 3 -

Rheumatology and Immunology,

Friedrich-Alexander-University

Erlangen-Nürnberg (FAU) and

University Hospital Erlangen, Erlangen,

Germany

Specialty section:

This article was submitted to Inflammation,

a section of the journal

Frontiers in Immunology

Received: 05 February 2019

Accepted: 05 June 2019

Published: 25 June 2019

Citation:

Sody S, Uddin M, Grüneboom A, Görgens A, Giebel B, Gunzer M and Brandau S (2019) Distinct

Spatio-Temporal Dynamics of

Tumor-Associated Neutrophils in

Small Tumor Lesions.

Front. Immunol. 10:1419.

doi: 10.3389/fimmu.2019.01419

\section{Distinct Spatio-Temporal Dynamics of Tumor-Associated Neutrophils in Small Tumor Lesions}

\author{
Simon Sody ${ }^{1}$, Mohib Uddin ${ }^{2}$, Anika Grüneboom ${ }^{3 \dagger}$, André Görgens ${ }^{4,5}$, Bernd Giebel ${ }^{4}$, \\ Matthias Gunzer ${ }^{3}$ and Sven Brandau ${ }^{1 *}$ \\ ${ }^{1}$ Department of Otorhinolaryngology, University Hospital Essen, University Duisburg-Essen, Essen, Germany, ${ }^{2}$ Respiratory \\ Global Medicines Development (GMD), AstraZeneca, Gothenburg, Sweden, ${ }^{3}$ Institute for Experimental Immunology and \\ Imaging, University Hospital Essen, University Duisburg-Essen, Essen, Germany, ${ }^{4}$ Institute for Transfusion Medicine, \\ University Hospital Essen, University Duisburg-Essen, Essen, Germany, ${ }^{5}$ Department of Laboratory Medicine, Karolinska \\ Institutet, Stockholm, Sweden
}

Across a majority of cancer types tumor-associated neutrophils (TAN) are linked with poor prognosis. However, the underlying mechanisms, especially the intratumoral behavior of TAN, are largely unknown. Using intravital multiphoton imaging on a mouse model with neutrophil-specific fluorescence, we measured the migration of TAN in distinct compartments of solid tumor cell lesions in vivo. By longitudinally quantifying the infiltration and persistence of TAN into growing tumors in the same animals, we observed cells that either populated the peripheral stromal zone of the tumor (peritumoral TAN) or infiltrated into the tumor core (intratumoral TAN). Intratumoral TAN showed prolonged tumor-associated persistence and reduced motility compared to peritumoral TAN, whose velocity increased with tumor progression. Selective pharmacological blockade of CXCR2 receptors using AZD5069 profoundly inhibited recruitment of TAN into peritumoral regions, while intratumoral infiltration was only transiently attenuated and rebounded at later time points. Our findings unravel distinct spatial dynamics of TAN that are partially and differentially regulated via the CXCR2 signaling pathway.

Keywords: tumor-associated neutrophils, neutrophil granulocytes, intravital imaging, multiphoton microscopy, tumor microenvironment, tumor immunology, CXCR2, AZD5069

\section{INTRODUCTION}

During the last decade, a high intratumoral frequency of tumor-associated neutrophils (TAN) was established as a strong predictor of poor clinical outcome in the majority of solid tumor entities (1-3). In fact, in a recent transcriptomic analysis of $\sim 18.000$ human tumor samples from 14 solid tumors neutrophils (secondary to mast cells) showed the strongest correlation with adverse cancer outcomes (1).

Despite this well-established prognostic role in the clinical setting, the mechanisms underlying a disease-promoting activity of neutrophils are still poorly understood. Murine studies have identified a variety of neutrophil-mediated pro-tumorigenic factors (4). Production of neutrophil-derived matrix metalloproteinases like MMP-9 were shown to release VEGF-A from the extracellular matrix (ECM) and thereby constitute a major source of pro-angiogenic factors in the tumor microenvironment (5-8). In addition, neutrophils can influence invasiveness and metastatic potential of tumor cells by angiotropism (9) or neutrophil granule-derived enzymes, 
that actively remodel the ECM and mutually activate tumoral proteases to promote the invasion of tumor cells (10). Priming of lung pre-metastatic niches through neutrophil MMPs enhanced the metastatic spread of mammary tumors (11). Likewise, human neutrophils, after CXCR2-dependent recruitment (12), and MAPK activation have the ability to induce multiple tumor promoting mechanisms (6), which includes the cortactinmediated induction of tumor cell invasion and metastasis in patients (13). Further, immunosuppressive neutrophils with myeloid-derived suppressor cell activity (PMN-MDSC) can drive tumor immune evasion (14-16).

Despite this important role of neutrophils in malignant disease, until recently, even in murine models, mechanistic studies on the recruitment and intratumoral biology of TAN were limited to histological tissue analyses, ex vivo investigations or depletion of neutrophils by antibodies without a direct observation of the live cells within the tumor. To a large extent, this has been based on existing models such as lys-EGFP, c-fmsEGFP, and hMRP8-Cre that were not neutrophil specific and hence also included the analysis of "contaminating" cells from the myelomonocytic and dendritic lineages (17-19). As such, immune-mediated mechanisms of neutrophil recruitment to the sites of tumor are incompletely understood.

Experimental murine studies and clinical correlation analyses have identified ligands for CXCR2 as major drivers of TAN recruitment into tumor lesions, involving CXCL1/KC, CXCL2/MIP-2, CXCL5/LIX, CXCL6, and MIF (12, 20-23). Consequently, at least in murine models, many of the diseasepromoting effects of neutrophils can be attenuated by CXCR2 blockade (24-26). In contrast to human neutrophils, where CXCR1 and CXCR2/IL-8 interaction is a major chemoattractant (27), in mice, CXCR1 has a redundant capacity for neutrophil trafficking whilst playing a predominant role in regulating degranulation (28). Neutrophil effector functions and trafficking to tissues are also context-dependent. While neutrophils were initially considered as purely pathogen-clearing innate effector cells, to date, complex and adaptable functions in infection, inflammation and cancer are emerging $(29,30)$.

In this study, we used AZD5069 to modulate recruitment of TAN into tumor lesions in vivo. AZD5069 is a small molecule antagonist with over 100 -fold selectivity for CXCR2 relative to CXCR1 receptor, that does not adversely affect neutrophilmediated host immunity $(31,32)$. Beyond a potential immunooncological target (20), AZD5069 has been extensively studied as an orally active immunotherapy in chronic respiratory diseases, including $\operatorname{COPD}(33,34)$, bronchiectasis (35) and severe asthma $(36,37)$. In murine tumor models CXCR2 blockade has been shown to modulate neutrophil trafficking to

\footnotetext{
Abbreviations: 2PM, two-photon microscopy; a.dest, distillated water; ADCC, antibody-dependent cellular cytotoxicity; BP, bandpass; COPD, chronic obstructive pulmonary disease; ECM, extracellular matrix; $\mathrm{FetCO}_{2}$, end-tidal $\mathrm{CO}_{2}$-fraction; G-MDSC, granulocytic myeloid-derived suppressor cells; HNC, head and neck cancer; HNSCC, head and neck squamous cell carcinoma; i.d., intradermal; i.p., intraperitoneal; i.v., intravenous; LP, longpass; MIP, maximum intension projection; NIR, near infrared; PBS, Phosphate buffered saline; SHG, second harmonic generation; TAN, Tumor-associated neutrophils; TME, Tumor microenvironment.
}

sites of chronic inflammation, subsequently reduced tumor and metastasis formation and enhanced treatment efficacy in distinct therapeutic conditions (38-41).

To directly image different phases of neutrophil invasion into locally growing tumors, we used a recently established mouse model, termed Catchup (42). This allowed us to uncover time-dependent changes in frequency, localization, and migratory patterns of neutrophils in small tumor lesions. We found that neutrophils localized in either intratumoral or peritumoral regions revealed distinct migratory patterns. Surprisingly, blockade of the CXCR2 chemokine receptors, previously believed to selectively inhibit migration of neutrophils into tumors and other inflammatory tissue lesions, was shown to markedly attenuate peritumoral stromal TAN, whilst only transiently blocking the recruitment of TAN into the early tumor cell lesion. These findings have important implications for the precision targeting of TAN in emerging cancer combination immunotherapies.

\section{MATERIALS AND METHODS}

\section{Animals}

All animal experiments were performed in accordance with the animal ethics committee of the state of North Rhine-Westphalia, Germany, and German guidelines for experimental animal welfare. Generation of Catchup ${ }^{\text {IVM-red }}$ was previously described (42). Catchup ${ }^{\text {IVM-red }}$ mice were bred in the animal facility of the Centre for Medical Biotechnology of the University Duisburg Essen and housed under specific pathogen-free conditions in individually ventilated cage racks. Both male and female Catchup ${ }^{\text {IVM-red }}$ mice were used at any age between 3 and 6 month, but animals were sex- and age-matched in each experiment as much as possible.

\section{Tumor Cell Culture}

The murine oropharyngeal cell line MOPC (C57BL/6-derived, HPV16 E6/E7 ${ }^{-}$) was kindly provided by J. Lee (Sanford Research/University of South Dakota, Sioux Falls, SD, USA) and cultured as described previously (43). MOPC ${ }^{\text {EGFP }}$ cells were generated by lentiviral gene transfer using a pCL6IEGwo empty vector (44) as previously described (45). Cells were washed twice in phosphate buffer saline (PBS) before injection into mouse dermis under sterile conditions.

\section{Tumor Model}

Syngeneic murine HNSCC line MOPC ${ }^{\text {EGFP }}$ were injected superficially in the dermis of the outer dorsal ear for intravital microscopy. Approximately 3 min before tumor cell injection the ear was depilated using commercially available depilatory cream, applied $<2 \mathrm{~min}$. Under Ketamin/Xylazin $(100 / 20 \mathrm{mg} / \mathrm{kg}$ body weight) anesthesia $\sim 10 \mu \mathrm{l}$ of $20^{*} 10^{6} / \mathrm{ml}$ cell suspension in PBS was injected into the ear dermis using a 30 -gauge cannula.

\section{Contralateral Day 3 Tumor}

In one experiment, on day 3 after tumor cell inoculation, AZD5069 and vehicle-treated Catchup ${ }^{\text {IVM-red }}$ mice were injected with a second tumor into the contralateral outer ear 
dermis. Tumor injection and CXCR2 blockade was performed as described below.

\section{CXCR2 Antagonism}

CXCR2 was blocked with small molecule antagonist AZD5069, which was provided by AstraZeneca. AZD5069 was diluted in vehicle solution consisting of $1.14 \% \mathrm{w} / \mathrm{w}$ HP- $\beta$-cyclodextrin and $0.5 \%$ Hydroxy propyl methyl cellulose (HPMC $6 \mathrm{cps}$ ) in $0.1 \mathrm{mM}$ Carbonate buffer ( $\mathrm{pH}$ 9.5-10). One-hundred microliter of $10 \mathrm{mM}$ AZD5069 or vehicle solution only (for control group) was continuously administered twice daily ( $12 \mathrm{~h}$ interval) via oral gavage starting $12 \mathrm{~h}$ before tumor cell injection.

\section{Intravital 2-Photon Microscopy}

Imaging of MOPC ${ }^{\mathrm{EGFP}}$ tumors in the outer dermis of the dorsal ear was performed non-invasively on costume build water heated aluminum stage. The ear was gently mounted on pre-warmed aluminum block using Vaseline, covered with a cover slip and encumbered with a $1 \mathrm{~cm}$ diameter metal ring. PBS was used as immersion medium beneath and above cover slip sealed with Vaseline to prevent drain. Long term anesthesia was controlled via intubation narcosis and mechanical ventilation (1.5\% Isoflurane in $\mathrm{O}_{2}$ ) using capnography to maintain physiologic ventilation (expiratory $\mathrm{CO}_{2}: \sim 20 \mathrm{mmHg}$ ). Mice were injected with $10 \mu \mathrm{L}$ of a $1 \mathrm{mM}$ QTracker ${ }^{\circledR} 655$ (Life Technologies, Darmstadt, Germany) solution i.v. to visualize blood vessels before constant observation in a Leica TCS SP8 MP microscope (Leica Microsystems, Mannheim, Germany) with simultaneous detection via hybrid-reflected light detectors and photomultiplier tubes with a HCX IRAPO L253/0.95 water objective. If not otherwise indicated excitation was performed at $960 \mathrm{~nm}$ using a Coherent Chameleon Vision II Ti:Saph-Laser (Coherent LaserSystems, Göttingen, Germany). The following filter settings were used: collagen (second harmonic generation, SHG) BP485/30; neutrophils (tdTomato transgene) BP585/50, blood vessels (QTracker $\left.{ }^{\circledR}\right)$ BP660/30, tumor cells (EGFP) BP525/50. Raw data were reconstructed with Imaris (Bitplane, Zurich, Switzerland) for quantitative analysis and generation of representative pictures and videos.

\section{Statistical Analysis}

Data were analyzed using GraphPad Prism Software (GraphPad Software, Inc., La Jolla, CA, USA). Statistical significance was assessed with paired or unpaired two-tailed $t$-test for the comparison of two groups and two-way ANOVA with Bonferroni post-tests for the comparison of multiple groups if not otherwise indicated. Results were considered statistically significant at $p \leq 0.05$.

\section{RESULTS}

\section{Establishment of a Longitudinal Intravital Imaging System to Monitor TAN Mobility and Migration During Early Engraftment of Tumor Cells}

At first, we established technical requirements crucial for high quality, unperturbed, longitudinal imaging of TAN in early tumor cell lesions. Maintaining body temperature is important for preserving normal physiology of mice during prolonged and longitudinal imaging. Common heating pads are unsuitable for this purpose, since periodical heating leads to relevant material expansion and contraction with enormous shifts in $\mathrm{z}$ direction. To circumvent this problem, we designed a water heated aluminum stage with an external heating unit, which was constantly perfused with $36^{\circ} \mathrm{C}$ warm water. After narcosis, depilation of the ear, tumor cell injection and i.v. blood labeling the mouse ear was gently mounted with petroleum jelly (Vaseline ${ }^{\circledR}$ ) in prone position on pre-warmed aluminum block and covered with a glass cover slip. For stable long-term imaging conditions $(>1 \mathrm{~h})$ endotracheal intubation and controlled isoflurane narcosis $\left(1.5 \%\right.$ Isoflurane in $100 \% \mathrm{O}_{2}$ ) with constant capnometry $\left(\mathrm{FetCO}_{2}=2-3 \%\right)$ proved successful. Continuous capnometry ensured appropriate ventilation, sufficient narcosis depth, tolerability and an adjusted recovery phase. For shortterm imaging periods $(<1 \mathrm{~h})$ i.p. ketamine narcosis without endotracheal intubation was sufficient. Using these procedures intravital imaging was performed on days 0,3 , and 6 (Figure 1A).

To this end, following the adoptive transfer of $\sim 150,000$ cells of the HNC cell line MOPC ${ }^{\mathrm{EGFP}}$ (45), an appropriate superficial tumor cell lesion was identified with epifluorescence and navigation through oculars. The autofluorescence of epidermal cells followed by overlay with the second harmonic generation (SHG) signal of the basal membrane during multiphoton acquisition permitted navigation through skin layers (Figure 1B). Mean size of the lesion analyzed inside the field of view increased over time from $\sim 0.007 \mathrm{~mm}^{3}$ (day 0 , $120-180 \mathrm{~min}$ after injection) to $0.017 \mathrm{~mm}^{3}$ (day 6) (Figure 1C). Within the tumor cell lesion, we identified TAN in two distinct regions relative to the tumor cell mass. The center of a compact tumor lesion, consisting of densely packed tumor cells, was considered intratumoral and TAN localized in this area were designated intra-TAN. The directly adjacent, SHG signal/collagen rich, area within the field of view was termed peritumoral compartment. The peritumoral compartment was defined as a maximum distance of $250 \mu \mathrm{m}$ from the tumor margin, which was expected to be in reach of paracrine tumoral conditioning factors, but without direct tumor cell contact (Figure 1D; Supplementary Video 1). TANs in this region were termed peri-TAN. Using our model, we could routinely record longitudinal sessions of TAN imaging in single tumor lesions from day 0 (up to $3 \mathrm{~h}$ post tumor cell injection) until days 3 and 6 post injection (Figure 1E).

This experimental model therefore has provided a reliable method for longitudinal monitoring of unmanipulated TAN in small newly established tumor cell lesions with high resolution and in the context of two different spatial compartments of the tumor microenvironment.

\section{Dynamics of Early Neutrophil Infiltration Into the Tumor Lesion}

Due to their small size, very early tumor lesions are not readily accessible to classical histological preparation and analysis. Hence, intravital 2PM was especially suited to monitor 
A Intravital 2-Photon microscopy

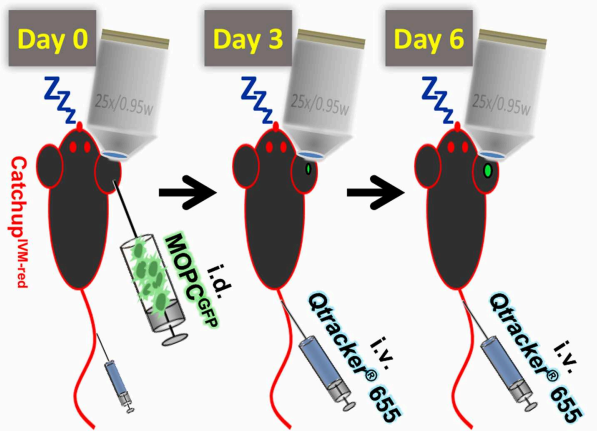

C

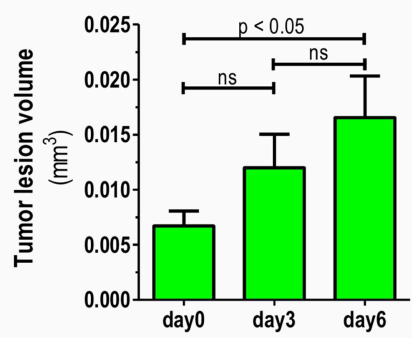

B

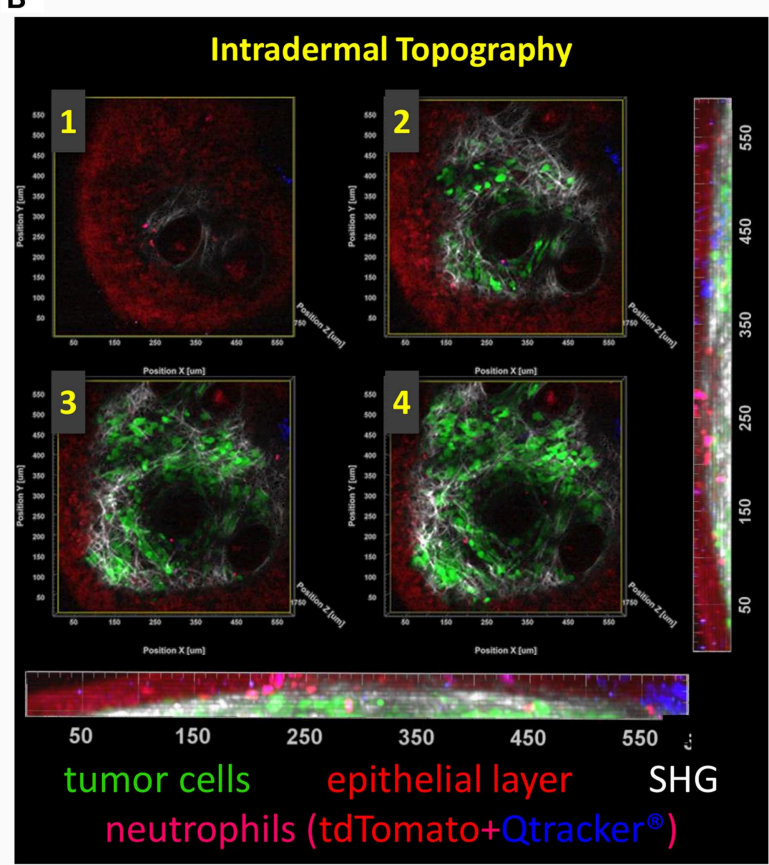

D

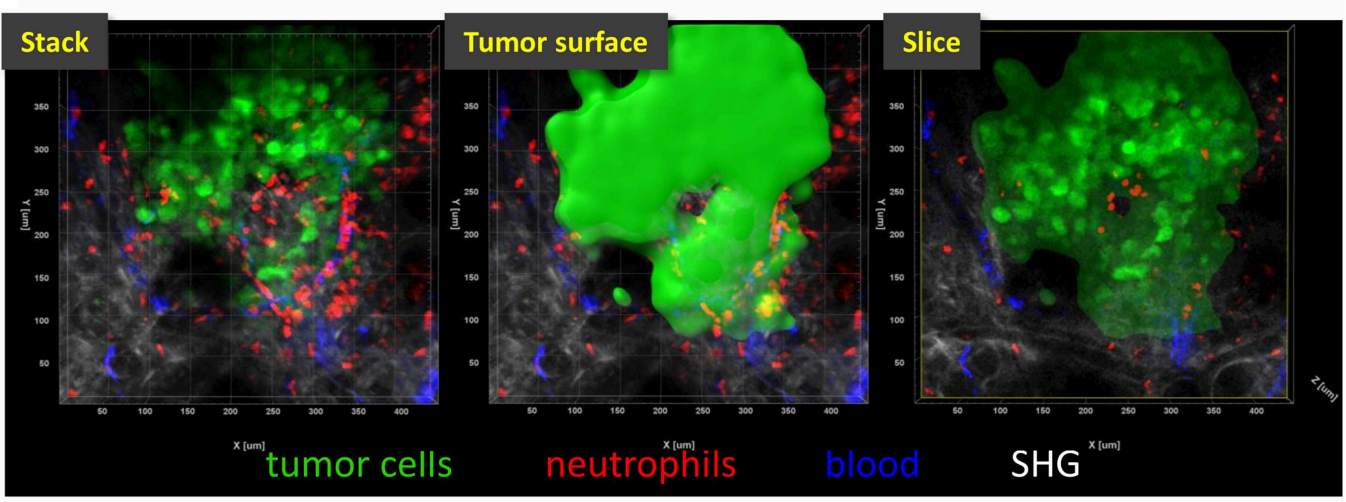

E

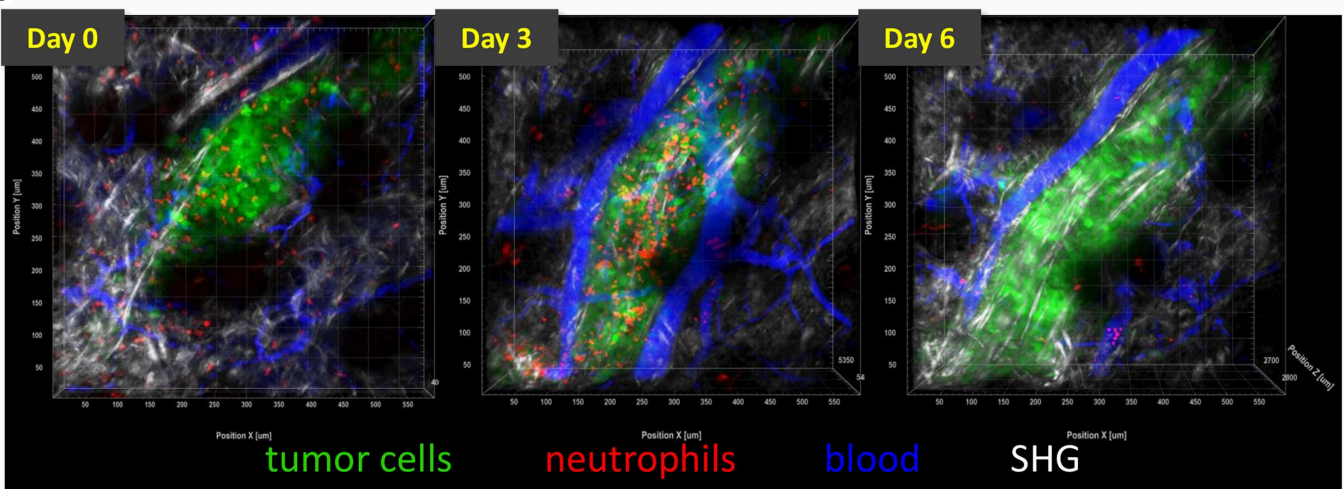

FIGURE 1 | Topography of small intradermal lesions of tumor cells. (A) Experimental scheme for longitudinal analysis of spatio-temporal dynamics of TAN. After

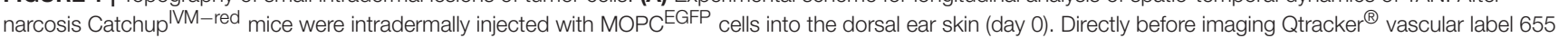
was injected i.v. into the tail vein or retrobulbar to visualize blood vessels. Imaging procedure on the same tumor lesion was repeated on days 3 and 6 . (B) Images $1-4$ show single cross sections in different depth in a multidimensional 2-Photon stack of an advanced tumor (>day 10). Orthogonal maximum intension projection (MIP) in the $x$-z-plane (bottom) and y-z-plane (right). Epidermal layer shows a weak autofluorescent signal in the tdTomato emission spectrum (red). Neutrophils show highly 
FIGURE 1 | fluorescent tdTomato signals and weakly accumulate Qtracker ${ }^{\circledR}$ over time (here 1.5 h post-injection is shown). After fluorescence overlay, neutrophils are displayed in magenta. This enables additional differentiation from autofluorescence by mixed color. Only residual Qtracker ${ }^{\circledR}$ (blue) from leakage or clotting and no blood staining is visible since $i . v$. injection occurred $1.5 \mathrm{~h}$ prior to image acquisition in this case. MOPCEGFP tumor cells are in green. Collagen fibers of the basal membrane and dermal matrix are visualized through SHG signal (white). Image 1 represents the epidermal-basal membrane border. (C) Means (+/- SEM) of tumor lesion volumes at different time points after tumor cell injection. Day $0=120-180$ min after tumor cell injection. $N=6$ animals. (D) Definition of tumoral compartments. Tumor volume was assessed by semi-automated surface generation of tumor cells (solid green). TAN inside tumor surface area were termed "intratumoral," cells outside were designated as "peritumoral." Cross-section through tumor volume reveals intra- vs. peritumoral TAN. (E) Intravital multidimensional 2-Photon images of representative tumor cell lesions in MIP from days 0 (120 min after tumor cell injection), 3, and 6 are depicted. 3D reconstruction was performed with Imaris ${ }^{\circledR}$ (Bitplane).

immune cell dynamics in these very early tumor cell lesions. Supplementary Video 2 records TAN infiltration between 45 and $120 \mathrm{~min}$ after tumor cell injection. At $60 \mathrm{~min}$ post injection, high numbers of highly migratory neutrophils started to infiltrate the tumor lesion (Supplementary Video 2). This influx followed sigmoid kinetics over the first $3 \mathrm{~h}$ (Figure $2 \mathrm{~A}$ ) and at $3 \mathrm{~h}$ post injection substantial numbers of neutrophils infiltrated the tumor injection site. In order to test whether the injection procedure itself may cause accumulation and recruitment of neutrophils we monitored injections of PBS (Figure 2E). While PBS injection indeed stimulated the recruitment of a smaller number of neutrophils, this influx was clearly low-level compared to the tumor cell-induced recruitment (Figures 2B,E). In addition, neutrophils, induced by this initial mechanical stimulus, showed short persistence and almost completely disappeared from the injection site by days 3 and 6 (Figures 2C-E).

We next investigated numbers and migration of individual TAN. While intra-TAN were mostly in contact with the carcinoma cells themselves, peri-TAN were in contact with the surrounding normal or stromal tissue and the extracellular matrix. At $2-3 \mathrm{~h}$ post injection, a substantial number of neutrophils was present in both compartments. At day 3 the frequency of peri-TAN was already strongly decreased from 322 cells $/ 0.015 \mathrm{~mm}^{3}$ (day 0 ) to 79 cells $/ 0.015 \mathrm{~mm}^{3}$, with further reduction by day 6 (Figures 2B-D). In contrast, intraTAN frequencies remained at high levels until day 3 and only decreased to lower levels by day 6 (Figures 1B, 2B-D).

We next quantified and compared the motility of TAN in these two compartments. At day 0, both intra-TAN and periTAN were highly migratory displaying an average velocity of 8.8 $\mu \mathrm{m} / \mathrm{min}$. This velocity was comparable to neutrophils recruited in response to PBS injection, suggesting that tumor cells strongly increased recruitment of neutrophils over the injection trigger as such (Figure 2B), but did not further modulate their speed (Figure 2F). We did not compare migratory properties of intravs. peri-TAN at day 0 , since neutrophils rapidly interchanged between compartments at this early point in time, making a clear allocation impossible. Instead the comparison of velocity of intra-TAN and peri-TAN was performed starting at day 3 and then followed up for at least 10 additional days. We observed that the velocities of intra-TAN strongly decreased during tumor development and TAN in larger developed tumors (day 14 or later) displayed a rather sessile phenotype (Figures 2G-I and Supplementary Video 3). Interestingly, and in contrast to directly tumor cell-associated neutrophils, peri-TAN increased their velocity over time. By day 14, this resulted in a substantial difference in velocity of intra- vs. peritumoral TAN (Figure 2I).
Next, we analyzed the directionality of TAN migration over time. At day 0 ( 1 to $3 \mathrm{~h}$ after injection) the infiltration of neutrophils into the lesion was directional (Figure 2J, directionality $>$ $0.5)$. In contrast, at days 3 and 6 , the overall directionality of TAN decreased over time, with peritumoral TAN constantly displaying a slightly higher directionality than intratumoral TAN (Figures 2K,L). Supplementary Video 4 supports this finding.

In conjunction, these data establish previously unknown timedependent differences in recruitment, persistence and migratory behavior of TAN located in either the intratumoral or the peritumoral area of the tumor microenvironment.

\section{Effect of CXCR2 Blockade on TAN Recruitment Into Tumors}

We have previously shown that TAN in this MOPC tumor model express high amounts of CXCR2 on their surface (45). Expression of CXCR2 ligands in the tumor microenvironment is believed to be a major pathway of TAN recruitment in murine tumor models $(20,46-48)$ and even in human HNC patients (3). Given the tumor-promoting role of TAN, interference with CXCR2/CXCR2-ligand interaction, has been proposed as a means to limit the pro-tumorigenic activity of TAN. Against this background, we investigated how CXCR2 blockade would affect frequencies and migratory patterns of TAN in this model. Consistent with this idea the small molecule CXCR2 antagonist, AZD5069 effectively blocked the influx of TAN into both the intratumoral and peritumoral areas at early time points after tumor cell inoculation (left columns, 2-3h, Figures 3A,B). However, unexpectedly, intratumoral TAN rebounded by days 3 and 6 despite AZD5069 treatment. Thus, CXCR2 blockade was unable to limit the recruitment of intra-TAN to intratumoral areas at days 3 and 6 (Figure 3A, compare Figure 3D for the respective still images of videos). This inability was in contrast to the durable inhibitory effect of AZD5069 on the frequency of peri-TAN, which did not show a significant rebound (Figure 3B). In fact, in most experiments CXCR2 blockade still maintained peri-TAN density to levels below 70 cells $/ 0.015$ $\mathrm{mm}^{3}$ on days 3 and 6 (Figure $3 \mathbf{B}$ ) while intra-TAN reached levels comparable to or even higher than control mice by day 3 and later (Figure 3A). This rebound of intra-TAN occurred despite reduced levels of circulating neutrophils in AZD5069treated mice until day 6 (Supplementary Figure 1). To confirm that AZD5069 was still generally active at day 3, we injected a second tumor at the contralateral side at this time point (Figure 3C). In this tumor, CXCR2 blockade still effectively inhibited the immediate recruitment of TAN into the tumor lesion at $2-3 \mathrm{~h}$ post injection. This indicates the in vivo activity 

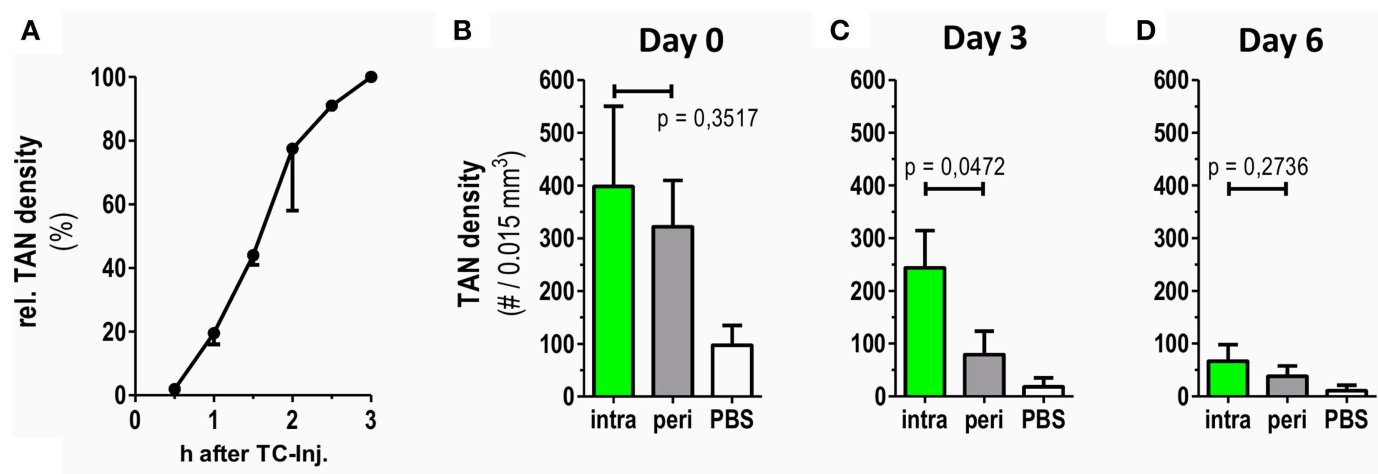

E

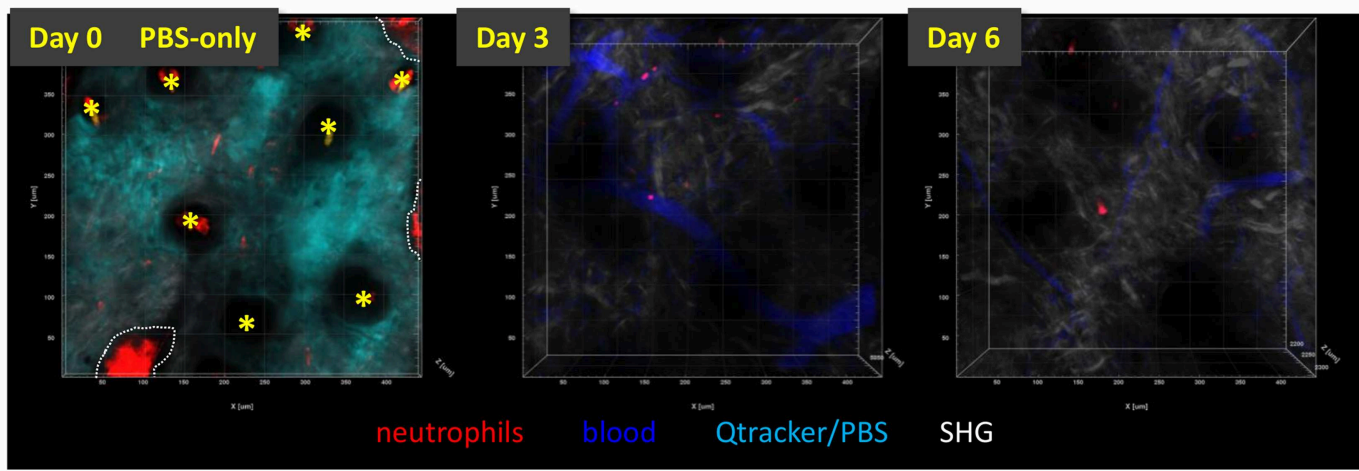

$\mathbf{F}$

G

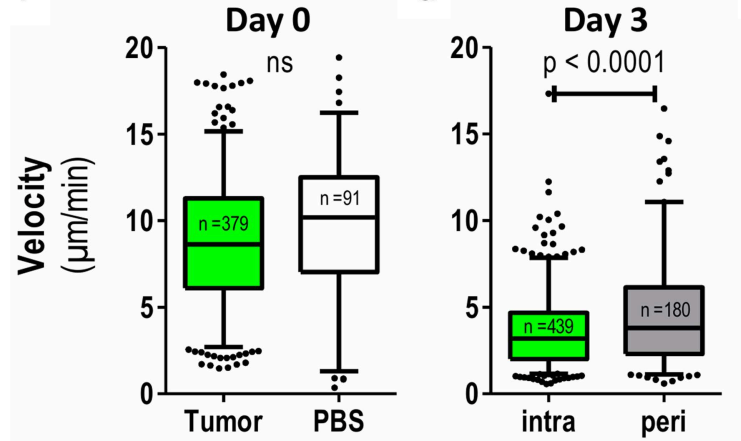

H
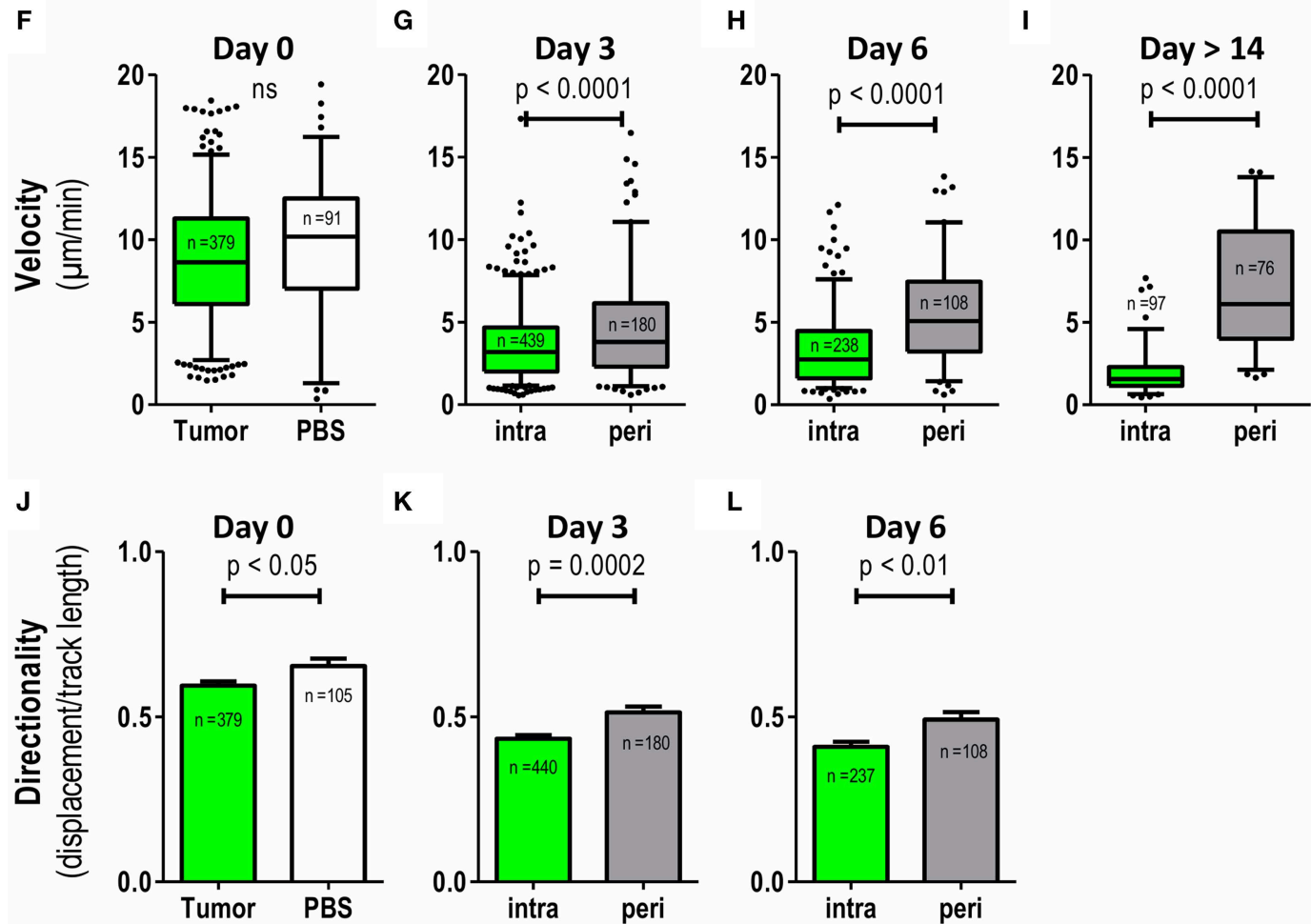

FIGURE 2 | Differential recruitment and migratory patterns of intratumoral vs. peritumoral neutrophils. Catchup ${ }^{\text {IVM-red }}$ mice were injected with MOPCEGFP cells into the dorsal ear dermis. TAN infiltration and motility was assessed by transdermal intravital 2PM. (A) Time course of TAN recruitment during the first $3 \mathrm{~h}$ after tumor cell inoculation. TAN density at $3 \mathrm{~h}$ was defined as 100\% (mean with SEM of 2 representative mice is shown). (B-D) Neutrophil densities were quantified in intratumoral (green bars) and peritumoral (gray bars) compartment and after PBS injection only (white bars) at days 0,3 , and 6 and depicted as number of TAN per 0.015 mm ${ }^{3}$ tumor tissue volume ( $n=6$ mice for tumor and $n=2$ mice for PBS). (E) Representative 2-D still images generated from three-dimensional multiphoton images in 
FIGURE 2 | maximum intension projection (MIP) demonstrating low neutrophil recruitment and persistence in control Catchup IVM-red mice injected with PBS+Qtracker ${ }^{\circledR}$ at dayO. At day 0 the Qtracker dye (+PBS) (cyan) was injected into the ear dermis only. On day 3 and 6 blood vessels were labeled by i.v. Qtracker ${ }^{\circledR}$ (blue) injection. Yellow asterisks mark hair follicles (day 0). Dotted white line indicate areas of invaginated epidermal layer (wrinkle) with high red autofluorescence. Scattered neutrophils are represented by cell-associated red signals in the tissue parenchyma. (F-I) Velocities of neutrophils in the tumor compartments assessed by semi-automated tracking. (J-L) Migration of TAN in (F-H) was also analyzed for directionality (track length/displacement). (F-L) $n=4$ for tumor bearing mice, $n=2$ for PBS, $n=3$ in (I) and cumulative number of analyzed single neutrophils depicted in each plot. Statistical significance was assessed with paired $t$-test (B-D) and unpaired two-tailed $t$-test $(\mathbf{F}-\mathbf{L}), \alpha=0.05$. Mean $+/-$ SEM is shown in bar graphs and 5-95\% percentiles in boxplots. 3D reconstruction, quantification and tracking were performed with Imaris $^{\circledR}$ (Bitplane).

of the compound despite the inability to exclude intra-TAN from tumors injected 3 days earlier in the same animal. Strikingly, also in the secondary tumor, AZD5069 lost effects on intraTAN 3 days post injection (Figure 3C) suggesting that this effect is mediated by a change in tumor biology rather than TAN biology. Consistent with observations in primary tumors, in most experiments CXCR2 blockade retained its inhibitory activity on the recruitment of peri-TAN by 3 days post injection also for the 2nd challenge tumor.

Having analyzed the effects of CXCR2 blockade on TAN densities, we then assessed the effect of CXCR2 blockade on fundamental motility parameters of TAN in both compartments. In day 0 intravital imaging showed the trafficking of neutrophils through vasculature directly adjacent to tumor cell lesions. As expected, also signs of neutrophil adhesion and rolling could be observed. Importantly, and confirming data from Figures 3A,B, the extravasation of TAN from vessels into the tumor lesion was completely abrogated by CXCR2 blockade at this time point (Supplementary Video 5). Since AZD5069 treatment completely prevented TAN recruitment to tumor lesions at the day of injection, motility was subsequently only analyzed at days 3 and 6 post injection. Interestingly, and despite its inability to reduce the recruitment of TAN to the intratumoral lesion at this time point (Figure 3A), AZD5069 still significantly reduced the motility of intra-TAN at day 3 (Figure 3E). However, at day 6 this effect of CXCR2 blockade on intra-TAN over vehicle treated animals was lost. A similar pattern of response was found for AZD5069 effects on the small numbers of peri-TAN. As depicted in Figure 3F, AZD5069 reduced the velocity of peri-TAN only at day 3 and not at day 6. Instead, the small number of peri-TAN, which infiltrated the tumors in the presence of CXCR2 blockade at day 6, seemed to display substantial mobility with a mean velocity above 5 $\mu \mathrm{m} / \mathrm{min}$ (Figure 3F). In summary, the effects of CXCR2 blockade on the migration demonstrated clear differences between intraand peri-TAN. In contrast, we observed a coherent effect of CXCR2 blockade on the directionality of TAN migration in both compartments. Here, AZD5069 treatment equally increased the directionality of TAN migration in both compartments (Figures 3G,H).

These data show, that, in addition to its distinct inhibitory effects on intra- and peri-TAN recruitment, AZD5069 also affects the intratumoral motility and directionality of these TAN subtypes in small tumor lesions.

\section{DISCUSSION}

In this study, we demonstrate the establishment of an experimental system of unperturbed longitudinal tumor-associated neutrophil (TAN) observation in the living mouse. To this end, we used a tumor cell injection model in the murine ear dermis. While this model has many advantages in terms of the imaging technology, it also has apparent limitations. Notably, injection models, particularly with respect to early growth phases do not fully recapitulate the complex multi-component tumor-stroma available in selected chemically induced or transgenic models. Despite these limitations, syngeneic transplantation models are very frequently used for experimental research and important aspects of immunooncology are being investigated in such models $(45,47,49,50)$. In terms of in vivo imaging, many experimental models to date require major surgical intervention to make tumor lesions accessible to imaging technologies $(51,52)$. This constitutes a trauma with subsequent effects on immune cell infiltration and behavior. Our model utilizes a minimally invasive procedure allowing for longitudinal long-term observations of the invasion of unperturbed TAN into a growing tumor. Although artificial disruption of tissue integrity occurs during intradermal injection in this model, the degree of damage is comparable to human tumor-associated wounds and inflammation, which are actually induced by invasive malignant progression or iatrogenic biopsies and surgery $(53,54)$. Our model, therefore, recapitulates certain aspects of regular tumor development in patients with cancer. In addition, we investigated the stimulus by PBS injection itself. By comparing neutrophil dynamics in PBS-only lesions with tumor cell injection, we could show that tumor cells are the major source of neutrophil attraction and exclusively induce persistence in this model. Tumor lesions showed four times greater neutrophil densities than PBS lesions. Further, the neutrophil influx in PBS lesions was transient; and resolved to background by day 3 , while TAN recruitment was durable over $>6$ days of observation.

Interestingly, we observed the formation of densely packed areas of tumor cells within $3 \mathrm{~h}$ after tumor cell injection and tumor cells showed tight microscopic cell-contacts. It is tempting to speculate that the injection of tumor cells and the formation of dense tumor cell areas also influences the biology of the surrounding stromal tissue. Our intravital imaging shows effective triggering of TAN recruitment into what we designated "intratumoral" and "peritumoral" (surrounding stromal) areas. Thus, at these early time points, most likely both tumor cell-derived and stromal cell-derived factors trigger TAN recruitment $(22,23,55)$. At later time points, intra-TAN showed prolonged persistence and reduced motility, consistent with in vitro observations demonstrating recruitment and delayed apoptosis of neutrophils in response to tumor-derived factors $(12,56)$. 

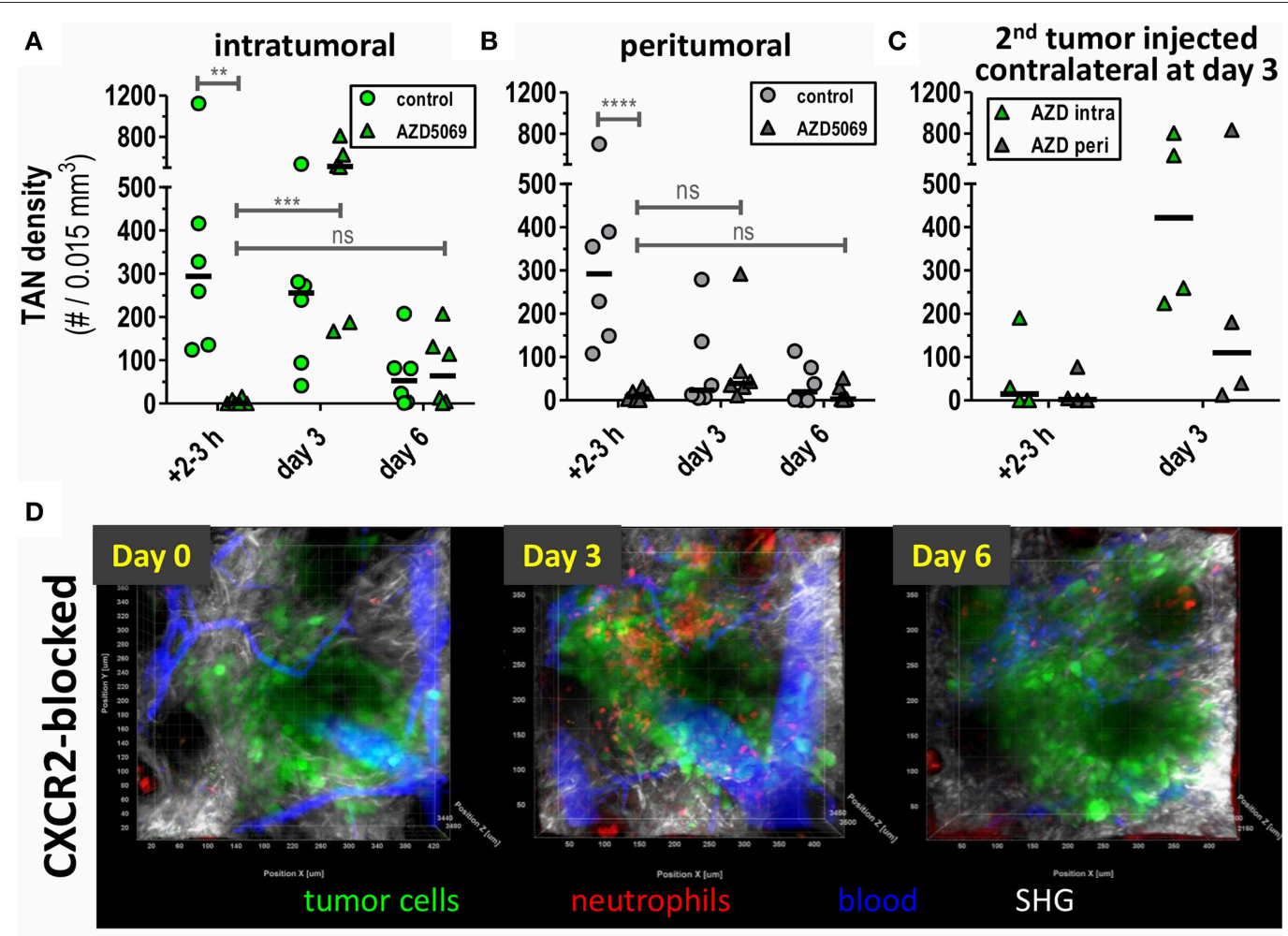

E

intratumoral

F

peritumoral
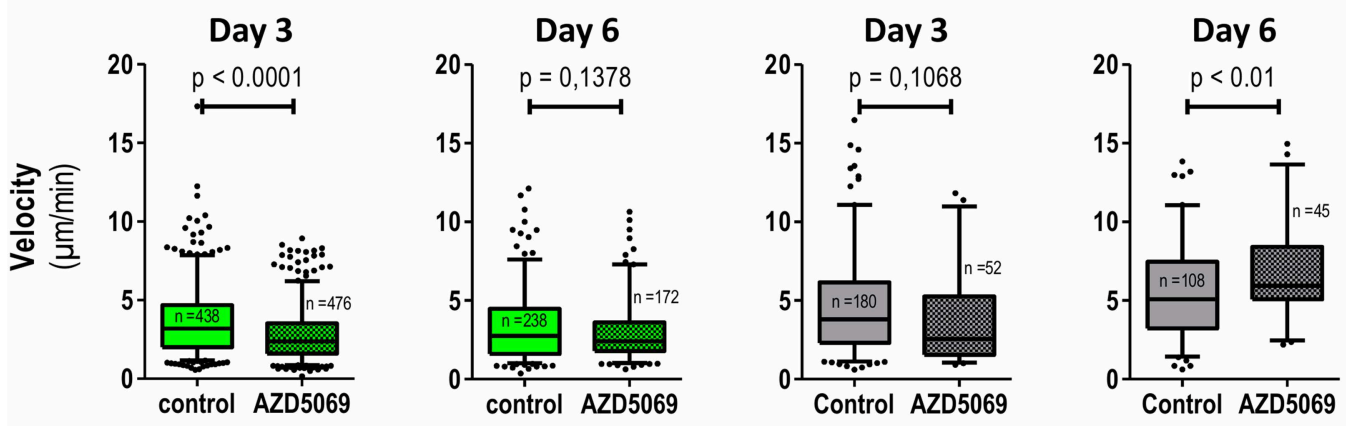

G

intratumoral

H

peritumoral
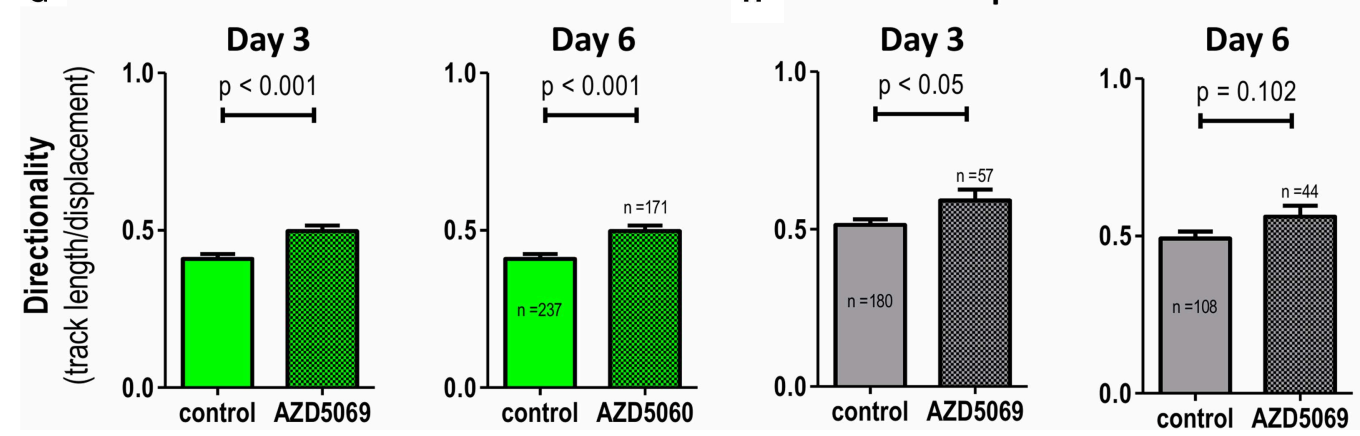

FIGURE 3 | Intratumoral and peritumoral TAN are differentially affected by CXCR2 blockade to AZD5069 treatment. CatchupIVM-red mice were injected with MOPCEGFP cells into the dorsal ear dermis. TAN infiltration and motility was assessed by transdermal intravital 2PM. To block CXCR2 AZD5069 was orally administered twice daily starting from day-1 before tumor cell inoculation until the end of the experiment. Intratumoral (A) and peritumoral (B) TAN infiltration was quantified as neutrophils per $0.015 \mathrm{~mm}^{3}$. Note the efficient reduction of both intratumoral and peritumoral TAN by AZD5069 at day 0. Also note the rebound of intratumoral, but not peritumoral, TAN under AZD5069 treatment at days 3 and 6. (C) Three days after injection of the primary tumor, AZD5069-treated mice received 
FIGURE 3 | a second tumor injection into the contralateral ear dermis. TAN density was determined in intra- and peritumoral areas $2-3 \mathrm{~h}$ and 3 days after 2 nd tumor injection. (D) Intravital multidimensional 2-Photon images of representative tumors of AZD5069 treated mice in maximum intension projection (MIP) on days 0, 3, and 6. Velocities and directionality of intratumoral $\mathbf{( E , G ) ~ a n d ~ p e r i t u m o r a l ~} \mathbf{( F , H ) ~ T A N ~ i n ~ A Z D 5 0 6 9 - t r e a t e d ~ c o m p a r e d ~ t o ~ c o n t r o l ~ m i c e ~ w e r e ~ d e t e r m i n e d ~ o n ~ d a y s ~} 3$ and 6 by intravital 2PM and semi-automated tracking (pooled data from $n=4$ mice per group). Statistical significance of difference was assessed with unpaired two-tailed $t$-test $(\alpha=0.05)$. Data in $(\mathbf{A}-\mathbf{C})$ are individual mice and the bar represents the median. Data in (E-F) are presented as box-plots with whiskers indicating the 5-95\% percentile. In (G-H) barplots of means with SEM. In (A-C) each symbol represents one mouse. ${ }^{\star \star} p<0.01,{ }^{\star \star \star} p<0.001,{ }^{\star \star \star \star} p<0.0001$, ns $=p>0.05 .3 D$ representation, quantification, and tracking were performed with Imaris ${ }^{\circledR}$ (Bitplane).

Most tumors consist of tumor cell islands and surrounding parenchyma or non-malignant stroma cells. Recently, we demonstrated a differential prognostic role of stromal vs. tumoral inflammation in head and neck squamous cell cancer (HNSCC) patients (57). Considering the emerging prognostic relevance of tumor-stroma constitution and sublocalization of immune infiltrates in solid tumors $(58,59)$, we especially focused on separate analyses of tumoral compartments in this study and could indeed find striking differences in TAN frequencies, motility and CXCR2-dependent regulation with regard to TAN localization.

A key finding was the reduced directionality and velocity of intra-TAN compared with peri-TAN. This reduced migratory activity of intra-TAN could be a possible reason for the persistence of high TAN densities in the intratumoral compartment beyond day 3 as opposed to the rapid decrease of peri-TAN frequencies. Additional evidence for the intratumoral persistence of intra-TAN comes from an analysis of adoptively transferred peripheral blood leukocytes from Catchup ${ }^{\text {IVM-red }}$ mice together with tumor cells into the ear dermis of C57BL/6 mice (Supplementary Figure 2). Here, we observed viable migrating adoptively transferred TAN until day 3 after transfer, suggesting that at least a certain number of intra-TAN can persist for up to 3 days in the tumor lesion. In contrast, the increased mobility of peri-TAN may lead to an increased chance of contact to distracting cues from sites away from the tumor lesion. It remains to be shown, which mechanisms are active in recruiting peri-TAN away from the tumor. Also delayed apoptosis of intra- vs. peri-TAN is a potential mechanism that might explain our findings $(56,60)$. New models utilizing photoactivatable GFP-transgenic neutrophils have recently been published (61) and could be used to further decipher the fate and function of intra-TAN after recruitment into the tumor core area.

CXCR2 is a major signaling pathway in neutrophil recruitment in tumors and non-malignant neutrophil-driven inflammatory diseases $(34,62-64)$. In a previous study, using the same MOPC cell line, we have demonstrated expression of $\mathrm{KC}$ and MIF by MOPC tumors (45). Interestingly, in the present study, we observed differential effects of CXCR2 blockade on TAN localized in either the intratumoral or peritumoral tissue areas. The fact that CXCR2 blockade efficiently blocked primary TAN influx on day 0 suggests a major role of the CXCR2 pathway in driving acute TAN recruitment. This is consistent with published intravital data in zebrafish larvae where neutrophil recruitment to wounds is abrogated by CXCR2 antagonists (65). However, in addition tumors may develop CXCR2 independent mechanisms of TAN recruitment which lead to stable TAN infiltration beyond day 3 . Alternatively, TAN residing in specialized niches of tumors might produce factors that recruit additional TANs, as has been shown recently for HGF, that is produced by TAN and recruits additional TANs via c-Met signaling (66). Similar observations have been made for neutrophils in necrotic lesions that induce a feed-forward loop for the recruitment of other neutrophils via leukotriene $\mathrm{B}_{4}$ (67). Future work needs to address, which mechanism is active in our model. Interestingly, in a model of zebrafish wounding additionally to the initial recruitment of neutrophils also the resolution of inflammation seemed to depend on CXCR2 (65).

The chemokine receptor CXCR2 is primarily expressed on mature neutrophils. However, in tumor hosts often an expansion of immature neutrophils occurs (45). Immature circulating neutrophils normally express low or no CXCR2. Evrard et al. recently showed that tumor bearing mice display elevated numbers of immature $\mathrm{CD} 101^{-}$neutrophils in blood and pancreatic tumors compared to naive mice (68). Those cells showed low surface expression of CXCR2. Interestingly, in this model $\mathrm{CXCR}^{-}$immature cells were still capable of normal tissue infiltration and interstitial migration. Hence, antagonizing CXCR2 using AZD5069 may selectively allow $\mathrm{CXCR}^{-}$immature neutrophils only to infiltrate transplanted tumors in our model. By contrast, CD101- immature neutrophils only account for 1 to $5 \%$ isolated neutrophils in blood and 5 to $16 \%$ of isolated neutrophils from pancreas in animals with low or high tumor burden, respectively (68). However, in our tumor model the numbers of intra-TAN in CXCR2-blocked Catchup ${ }^{\text {IVM-red }}$ mice even exceeded those of vehicle treated animals on day 3. In addition, using the same tumor cell line model as in this study, we recently reported that in both, naïve and tumor-bearing C57BL/6 mice, neutrophils in the blood, bone marrow and spleen consistently express considerable amounts of CXCR2 (45). The high TAN content under CXCR2 blockade occurs despite reduced systemic levels of circulating neutrophils (Supplementary Figure 1). Next to TAN-intrinsic mechanisms, it might still be possible, that the tumor changes its phenotype in a co-evolution with TAN or other infiltrating immune cells, which should be investigated in future studies. Furthermore, tumor cell triggered differential chemokine modifications in the densely packed intratumoral compartment may differ from chemokine processing and constitution in the peritumoral compartment. For neutrophil activating chemokines, it is known that their effect is modulated by post-translational changes like nitration or binding to glycosaminoglycans (69). Additionally, differential constitution in terms of extracellular matrix or extracellular proteolytic activity of both compartments may lead to differential conformational changes of the present chemokines to predominantly monomers or dimers. Since it was shown for 
CXCL1 that monomeric and dimeric form display differential activity in CXCR2 binding leading to a possible fine-tuning of chemokine-receptor pair effects (70), it is plausible that differential chemokine constitution in both compartments may lead to differential migratory properties of TAN.

In summary, our data represent the first targeted observation of unperturbed TAN in the living mouse during very early tumor establishment in vivo. Despite known and obvious limitations in terms of tumor cell development, our transplantable tumor model for intravital imaging reflects certain elements of human tumor cell biology and allows longitudinal tracking of spatiotemporal dynamics of unperturbed genetically labeled TAN. Clearly, our data suggest that infiltration of intratumoral lesion and peritumoral stroma are differentially regulated in terms of chemo-attractive and repulsive cues including the respective chemokine receptors and ligands involved. Interestingly, CXCR2 antagonism by AZD5069 is ineffective in preventing $\mathrm{Ly}_{6 \mathrm{G}}{ }^{+}$cell recruitment to tumor lesions at latter timepoints. Additional studies are required to decipher the complex bi-directional cross-talk of tumor tissue and TAN responsible for this dynamic interplay. Preliminary data in our group indicate strong tumor promoting features of intratumoral TAN (data not shown). Hence, our intravital system opens the possibility to further functionally characterize these distinct classes of TAN in different tumoral compartments and thereby enables unpreceded insight into TAN biology in the living animal.

\section{ETHICS STATEMENT}

This study was carried out in accordance with the recommendations of the animal ethics committee of the state of North Rhine-Westphalia, Germany, and German guidelines for experimental animal welfare. The protocol was

\section{REFERENCES}

1. Gentles AJ, Newman AM, Liu CL, Bratman SV, Feng W, Kim D, et al. The prognostic landscape of genes and infiltrating immune cells across human cancers. Nat Med. (2015) 21:938-45. doi: 10.1038/nm.3909

2. Shen M, Hu P, Donskov F, Wang G, Liu Q, Du J. Tumor-associated neutrophils as a new prognostic factor in cancer: a systematic review and meta-analysis. PLoS ONE. (2014) 9:e98259. doi: 10.1371/journal.pone.0098259

3. Trellakis S, Bruderek K, Dumitru CA, Gholaman H, Gu X, Bankfalvi A, et al. Polymorphonuclear granulocytes in human head and neck cancer: enhanced inflammatory activity, modulation by cancer cells and expansion in advanced disease. Int J Cancer. (2011) 129:2183-93. doi: 10.1002/ijc.25892

4. Coffelt SB, Wellenstein MD, de Visser KE. Neutrophils in cancer: neutral no more. Nat Rev Cancer. (2016) 16:431-46. doi: 10.1038/nrc.2016.52

5. Deryugina EI, Zajac E, Juncker-Jensen A, Kupriyanova TA, Welter L, Quigley JP. Tissue-infiltrating neutrophils constitute the major in vivo source of angiogenesis-inducing MMP-9 in the tumor microenvironment. Neoplasia. (2014) 16:771-88. doi: 10.1016/j.neo.2014.08.013

6. Dumitru CA, Fechner MK, Hoffmann TK, Lang S, Brandau S. A novel p38MAPK signaling axis modulates neutrophil biology in head and neck cancer. J Leukoc Biol. (2012) 91:591-8. doi: 10.1189/jlb.0411193

7. Kuang DM, Zhao Q, Wu Y, Peng C, Wang J, Xu Z, et al. Peritumoral neutrophils link inflammatory response to disease progression by fostering approved by the animal ethics committee of the state of North Rhine-Westphalia, Germany.

\section{AUTHOR CONTRIBUTIONS}

SB, SS, and MG: conceptualization. SS and AGr: investigation. SS, SB, MG, and MU: writing manuscript. SB, MG, and SS: data curation and analysis. SB, MU, MG, AGr, AGö, and BG: resource. SB, MG, MU, and AGö: reviewing manuscript.

\section{FUNDING}

This study was supported by the Else-Kröner Fresenius Foundation through the ELAN programme. The funding agency provided scholarship support and partly funded the consumables used in this study. The funder had no role in planning or executing the research.

\section{ACKNOWLEDGMENTS}

The authors gratefully acknowledge advice and technical support by Alexandra Brenzel and Anthony Squire from Imaging Center Essen (IMCES). We thank Anja Hasenberg for support with multiphoton imaging and animal management and Katrin Hahnel for preliminary work and advise. The authors would like to thank Dr Anna Malmgren (AstraZeneca, Gothenburg) for her support in providing the AZD5069 compound for exploratory research.

\section{SUPPLEMENTARY MATERIAL}

The Supplementary Material for this article can be found online at: https://www.frontiersin.org/articles/10.3389/fimmu. 2019.01419/full\#supplementary-material

angiogenesis in hepatocellular carcinoma. J Hepatol. (2011) 54:948-55. doi: 10.1016/j.jhep.2010.08.041

8. Nozawa H, Chiu C, Hanahan D. Infiltrating neutrophils mediate the initial angiogenic switch in a mouse model of multistage carcinogenesis. Proc Natl Acad Sci USA. (2006) 103:12493-8. doi: 10.1073/pnas.0601807103

9. Bald T, Quast T, Landsberg J, Rogava M, Glodde N, Lopez-Ramos $\mathrm{D}$, et al. Ultraviolet-radiation-induced inflammation promotes angiotropism and metastasis in melanoma. Nature. (2014) 507:109-13. doi: 10.1038/nature13111

10. Shamamian P, Schwartz JD, Pocock BJ, Monea S, Whiting D, Marcus SG, et al. Activation of progelatinase A (MMP-2) by neutrophil elastase, cathepsin $\mathrm{G}$, and proteinase-3: a role for inflammatory cells in tumor invasion and angiogenesis. J Cell Physiol. (2001) 189:197-206. doi: 10.1002/jcp.10014

11. Yan HH, Pickup M, Pang Y, Gorska AE, Li Z, Chytil A, et al. Gr$1+\mathrm{CD} 11 \mathrm{~b}+$ myeloid cells tip the balance of immune protection to tumor promotion in the premetastatic lung. Cancer Res. (2010) 70:6139-49. doi: 10.1158/0008-5472.CAN-10-0706

12. Dumitru CA, Gholaman H, Trellakis S, Bruderek K, Dominas N, Gu X, et al. Tumor-derived macrophage migration inhibitory factor modulates the biology of head and neck cancer cells via neutrophil activation. Int J Cancer. (2011) 129:859-69. doi: 10.1002/ijc.25991

13. Dumitru CA, Bankfalvi A, Gu X, Eberhardt WE, Zeidler R, Lang $\mathrm{S}$, et al. Neutrophils activate tumoral CORTACTIN to enhance 
progression of orohypopharynx carcinoma. Front Immunol. (2013) 4:33. doi: 10.3389/fimmu.2013.00033

14. Brandau S, Moses K, Lang S. The kinship of neutrophils and granulocytic myeloid-derived suppressor cells in cancer: cousins, siblings or twins? Semin Cancer Biol. (2013) 23:171-82. doi: 10.1016/j.semcancer.2013. 02.007

15. Gabrilovich DI, Ostrand-Rosenberg S, Bronte V. Coordinated regulation of myeloid cells by tumours. Nat Rev Immunol. (2012) 12:253-68. doi: 10.1038/nri3175

16. Lang S, Bruderek K, Kaspar C, Hoing B, Kanaan O, Dominas N, et al. Clinical relevance and suppressive capacity of human myeloidderived suppressor cell subsets. Clin Cancer Res. (2018) 24:4834-44. doi: 10.1158/1078-0432.CCR-17-3726

17. Abram CL, Roberge GL, Hu Y, Lowell CA. Comparative analysis of the efficiency and specificity of myeloid-Cre deleting strains using ROSA-EYFP reporter mice. J Immunol Methods. (2014) 408:89-100. doi: 10.1016/j.jim.2014.05.009

18. Faust N, Varas F, Kelly LM, Heck S, Graf T. Insertion of enhanced green fluorescent protein into the lysozyme gene creates mice with green fluorescent granulocytes and macrophages. Blood. (2000) 96:719-26.

19. Sasmono RT, Oceandy D, Pollard JW, Tong W, Pavli P, Wainwright BJ, et al. A macrophage colony-stimulating factor receptor-green fluorescent protein transgene is expressed throughout the mononuclear phagocyte system of the mouse. Blood. (2003) 101:1155-63. doi: 10.1182/blood-2002-02-0569

20. Acharyya S, Oskarsson T, Vanharanta S, Malladi S, Kim J, Morris PG, et al. A CXCL1 paracrine network links cancer chemoresistance and metastasis. Cell. (2012) 150:165-78. doi: 10.1016/j.cell.2012.04.042

21. Ji H, Houghton AM, Mariani TJ, Perera S, Kim CB, Padera R, et al. K-ras activation generates an inflammatory response in lung tumors. Oncogene. (2006) 25:2105-12. doi: 10.1038/sj.onc.1209237

22. Lazennec G, Richmond A. Chemokines and chemokine receptors: new insights into cancer-related inflammation. Trends Mol Med. (2010) 16:133-44. doi: 10.1016/j.molmed.2010.01.003

23. Vandercappellen J, Van Damme J, Struyf S. The role of CXC chemokines and their receptors in cancer. Cancer Lett. (2008) 267:226-44. doi: 10.1016/j.canlet.2008.04.050

24. Chao T, Furth EE, Vonderheide RH. CXCR2-dependent accumulation of tumor-associated neutrophils regulates T-cell immunity in pancreatic ductal adenocarcinoma. Cancer Immunol Res. (2016) 4:968-82. doi: 10.1158/2326-6066.CIR-16-0188

25. Highfill SL, Cui Y, Giles AJ, Smith JP, Zhang H, Morse E, et al. Disruption of CXCR2-mediated MDSC tumor trafficking enhances anti-PD1 efficacy. Sci Transl Med. (2014) 6:237-67. doi: 10.1126/scitranslmed.3007974

26. Moses K, Brandau S. Human neutrophils: their role in cancer and relation to myeloid-derived suppressor cells. Semin Immunol. (2016) 28:187-96. doi: 10.1016/j.smim.2016.03.018

27. Aul R, Patel S, Summerhill S, Kilty I, Plumb J, Singh D. LPS challenge in healthy subjects: an investigation of neutrophil chemotaxis mechanisms involving CXCR1 and CXCR2. Int Immunopharmacol. (2012) 13:225-31. doi: 10.1016/j.intimp.2012.04.008

28. Swamydas M, Gao JL, Break TJ, Johnson MD, Jaeger M, Rodriguez CA, et al. CXCR1-mediated neutrophil degranulation and fungal killing promote Candida clearance and host survival. Sci Transl Med. (2016) 8:322ra310. doi: 10.1126/scitranslmed.aac7718

29. Liew PX, Kubes P. The neutrophil's role during health and disease. Physiol Rev. (2019) 99:1223-48. doi: 10.1152/physrev.00012.2018

30. Mantovani A, Cassatella MA, Costantini C, Jaillon S. Neutrophils in the activation and regulation of innate and adaptive immunity. Nat Rev Immunol. (2011) 11:519-31. doi: 10.1038/nri3024

31. Jurcevic S, Humfrey C, Uddin M, Warrington S, Larsson B, Keen C. The effect of a selective CXCR2 antagonist (AZD5069) on human blood neutrophil count and innate immune functions. Br J Clin Pharmacol. (2015) 80:1324-36. doi: $10.1111 /$ bcp. 12724

32. Uddin M, Betts C, Robinson I, Malmgren A, Humfrey C. The chemokine CXCR2 antagonist (AZD5069) preserves neutrophil-mediated host immunity in non-human primates. Haematologica. (2017) 102:e65-8. doi: 10.3324/haematol.2016.152371
33. Kirsten AM, Forster K, Radeczky E, Linnhoff A, Balint B, Watz H, et al. The safety and tolerability of oral AZD5069, a selective CXCR2 antagonist, in patients with moderate-to-severe COPD. Pulm Pharmacol Ther. (2015) 31:36-41. doi: 10.1016/j.pupt.2015.02.001

34. Pedersen F, Waschki B, Marwitz S, Goldmann T, Kirsten A, Malmgren A, et al. Neutrophil extracellular trap formation is regulated by CXCR2 in COPD neutrophils. Eur Respir J. (2018) 51:1700970. doi: 10.1183/13993003.00970-2017

35. De Soyza A, Pavord I, Elborn JS, Smith D, Wray H, Puu M, et al. A randomised, placebo-controlled study of the CXCR2 antagonist AZD5069 in bronchiectasis. Eur Respir J. (2015) 46:1021-32. doi: 10.1183/13993003.00148-2015

36. O’Byrne PM, Metev H, Puu M, Richter K, Keen C, Uddin M, et al. Efficacy and safety of a CXCR2 antagonist, AZD5069, in patients with uncontrolled persistent asthma: a randomised, double-blind, placebo-controlled trial. Lancet Respir Med. (2016) 4:797-806. doi: 10.1016/S2213-2600(16)30227-2

37. Watz H, Uddin M, Pedersen F, Kirsten A, Goldmann T, Stellmacher F, et al. Effects of the CXCR2 antagonist AZD5069 on lung neutrophil recruitment in asthma. Pulm Pharmacol Ther. (2017) 45:121-3. doi: 10.1016/j.pupt.2017.05.012

38. Di Mitri D, Toso A, Chen JJ, Sarti M, Pinton S, Jost TR, et al. Tumourinfiltrating Gr-1+ myeloid cells antagonize senescence in cancer. Nature. (2014) 515:134-7. doi: 10.1038/nature13638

39. Gong L, Cumpian AM, Caetano MS, Ochoa CE, De la Garza MM, Lapid DJ, et al. Promoting effect of neutrophils on lung tumorigenesis is mediated by CXCR2 and neutrophil elastase. Mol Cancer. (2013) 12:154. doi: 10.1186/1476-4598-12-154

40. Jamieson T, Clarke M, Steele CW, Samuel MS, Neumann J, Jung A, et al. Inhibition of CXCR2 profoundly suppresses inflammationdriven and spontaneous tumorigenesis. J Clin Invest. (2012) 122:3127-44. doi: 10.1172/JCI61067

41. Steele CW, Karim SA, Leach JDG, Bailey P, Upstill-Goddard R, Rishi L, et al. CXCR2 inhibition profoundly suppresses metastases and augments immunotherapy in pancreatic ductal adenocarcinoma. Cancer Cell. (2016) 29:832-45. doi: 10.1016/j.ccell.2016.04.014

42. Hasenberg A, Hasenberg M, Mann L, Neumann F, Borkenstein L, Stecher $\mathrm{M}$, et al. Catchup: a mouse model for imaging-based tracking and modulation of neutrophil granulocytes. Nat Methods. (2015) 12:445-52. doi: 10.1038/nmeth.3322

43. Williams R, Lee DW, Elzey BD, Anderson ME, Hostager BS, Lee JH. Preclinical models of HPV+ and HPV- HNSCC in mice: an immune clearance of HPV+ HNSCC. Head Neck. (2009) 31:911-8. doi: 10.1002/hed. 21040

44. Leurs C, Jansen M, Pollok KE, Heinkelein M, Schmidt M, Wissler M, et al. Comparison of three retroviral vector systems for transduction of nonobese diabetic/severe combined immunodeficiency mice repopulating human CD34+ cord blood cells. Hum Gene Ther. (2003) 14:509-19. doi: 10.1089/104303403764539305

45. Moses K, Klein JC, Mann L, Klingberg A, Gunzer M, Brandau S. Survival of residual neutrophils and accelerated myelopoiesis limit the efficacy of antibody-mediated depletion of Ly-6G+ cells in tumor-bearing mice. J Leukoc Biol. (2016) 99:811-23. doi: 10.1189/jlb.1HI0715-289R

46. Jablonska J, Wu CF, Andzinski L, Leschner S, Weiss S. CXCR2-mediated tumor-associated neutrophil recruitment is regulated by IFN-beta. Int $J$ Cancer. (2014) 134:1346-58. doi: 10.1002/ijc.28551

47. Sharma B, Nannuru KC, Varney ML, Singh RK. Host Cxcr2-dependent regulation of mammary tumor growth and metastasis. Clin Exp Metastasis. (2015) 32:65-72. doi: 10.1007/s10585-014-9691-0

48. Singh S, Varney M, Singh RK. Host CXCR2-dependent regulation of melanoma growth, angiogenesis, and experimental lung metastasis. Cancer Res. (2009) 69:411-5. doi: 10.1158/0008-5472.CAN-08-3378

49. Fridlender ZG, Sun J, Kim S, Kapoor V, Cheng G, Ling L, et al. Polarization of Tumor-Associated Neutrophil (TAN) phenotype by TGF-?: "N1" versus "N2" TAN. Cancer Cell. (2009) 16:183-94. doi: 10.1016/j.ccr.2009.06.017

50. Klein JC, Moses K, Zelinskyy G, Sody S, Buer J, Lang S, et al. Combined tolllike receptor 3/7/9 deficiency on host cells results in T-cell-dependent control of tumour growth. Nat Commun. (2017) 8:14600. doi: 10.1038/ncomms14600 
51. Headley MB, Bins A, Nip A, Roberts EW, Looney MR, Gerard A, et al. Visualization of immediate immune responses to pioneer metastatic cells in the lung. Nature. (2016) 531:513-7. doi: 10.1038/nature16985

52. Lohela M, Casbon AJ, Olow A, Bonham L, Branstetter D, Weng N, et al. Intravital imaging reveals distinct responses of depleting dynamic tumor-associated macrophage and dendritic cell subpopulations. Proc Natl Acad Sci USA. (2014) 111:E5086-95. doi: 10.1073/pnas.1419899111

53. Antonio N, Bonnelykke-Behrndtz ML, Ward LC, Collin J, Christensen IJ, Steiniche T, et al. The wound inflammatory response exacerbates growth of pre-neoplastic cells and progression to cancer. EMBO J. (2015) 34:2219-36. doi: 10.15252/embj.201490147

54. Rosowski EE, Huttenlocher A. Neutrophils, wounds, and cancer progression. Dev Cell. (2015) 34:134-6. doi: 10.1016/j.devcel.2015.07.005

55. Del Prete A, Schioppa T, Tiberio L, Stabile H, Sozzani S. Leukocyte trafficking in tumor microenvironment. Curr Opin Pharmacol. (2017) 35:407. doi: 10.1016/j.coph.2017.05.004

56. Zhou SL, Zhou ZJ, Hu ZQ, Huang XW, Wang Z, Chen EB, et al. Tumor-associated neutrophils recruit macrophages and T-regulatory cells to promote progression of hepatocellular carcinoma and resistance to sorafenib. Gastroenterology. (2016) 150:1646-58 e1617. doi: 10.1053/j.gastro.2016.02.040

57. Höing B, Kanaan O, Altenhoff P, Petri R, Thangavelu K, Schluter A, et al. Stromal versus tumoral inflammation differentially contribute to metastasis and poor survival in laryngeal squamous cell carcinoma. Oncotarget. (2018) 9:8415-26. doi: 10.18632/oncotarget.23865

58. Feng Z, Bethmann D, Kappler M, Ballesteros-Merino C, Eckert A, Bell RB, et al. Multiparametric immune profiling in HPV- oral squamous cell cancer. JCI Insight. (2017) 2:93652. doi: 10.1172/jci.insight.93652

59. Mezheyeuski A, Bergsland CH, Backman M, Djureinovic D, Sjoblom T, Bruun J, et al. Multispectral imaging for quantitative and compartment-specific immune infiltrates reveals distinct immune profiles that classify lung cancer patients. J Pathol. (2017) 244:421-31. doi: 10.1002/path.5026

60. Andzinski L, Wu CF, Lienenklaus S, Kroger A, Weiss S, Jablonska J. Delayed apoptosis of tumor associated neutrophils in the absence of endogenous IFN-beta. Int J Cancer. (2015) 136:572-83. doi: 10.1002/ijc.28957

61. Wang J, Hossain M, Thanabalasuriar A, Gunzer M, Meininger C, Kubes P. Visualizing the function and fate of neutrophils in sterile injury and repair. Science. (2017) 358:111-6. doi: 10.1126/science.aam9690

62. Boppana NB, Devarajan A, Gopal K, Barathan M, Bakar SA, Shankar EM, et al. Blockade of CXCR2 signalling: a potential therapeutic target for preventing neutrophil-mediated inflammatory diseases. Exp Biol Med. (2014) 239:50918. doi: 10.1177/1535370213520110

63. Eash KJ, Greenbaum AM, Gopalan PK, Link DC. CXCR2 and CXCR4 antagonistically regulate neutrophil trafficking from murinebone marrow. J Clin Investig. (2010) 120:2423-31. doi: 10.1172/JCI 41649

64. Waugh DJ, Wilson C. The interleukin-8 pathway in cancer. Clin Cancer Res. (2008) 14:6735-41. doi: 10.1158/1078-0432.CCR-07-4843

65. Powell D, Tauzin S, Hind LE, Deng Q, Beebe DJ, Huttenlocher A Chemokine signaling and the regulation of bidirectional leukocyte migration in interstitial tissues. Cell Rep. (2017) 19:1572-85. doi: 10.1016/j.celrep.2017. 04.078

66. Glodde N, Bald T, van den Boorn-Konijnenberg D, Nakamura K, O’Donnell JS, Szczepanski S, et al. Reactive neutrophil responses dependent on the receptor tyrosine kinase c-MET limit cancer immunotherapy. Immunity. (2017) 47, 789-802 e789. doi: 10.1016/j.immuni.2017.09.012

67. Lammermann T, Afonso PV, Angermann BR, Wang JM, Kastenmuller W, Parent CA, et al. Neutrophil swarms require LTB4 and integrins at sites of cell death in vivo. Nature. (2013) 498:371-5. doi: 10.1038/nature 12175

68. Evrard M, Kwok IWH, Chong SZ, Teng KWW, Becht E, Chen J, et al. Developmental analysis of bone marrow neutrophils reveals populations specialized in expansion, trafficking, and effector functions. Immunity. (2018) 48:364-79. doi: 10.1016/j.immuni.2018.02.002

69. Thompson S, Martinez-Burgo B, Sepuru KM, Rajarathnam K, Kirby JA, Sheerin NS, et al. Regulation of chemokine function: the roles of GAGbinding and post-translational nitration. Int J Mol Sci. (2017) 18:E1692. doi: 10.3390/ijms18081692

70. Ravindran A, Sawant KV, Sarmiento J, Navarro J, Rajarathnam K. Chemokine CXCL1 dimer is a potent agonist for the CXCR2 receptor. J Biol Chem. (2013) 288:12244-52. doi: 10.1074/jbc.M112.443762

Conflict of Interest Statement: AGö is a consultant for and has equity interest in Evox Therapeutics Ltd., Oxford, UK. MU is an employee of AstraZeneca and holds shares in AstraZeneca.

The remaining authors declare that the research was conducted in the absence of any commercial or financial relationships that could be construed as a potential conflict of interest.

Copyright () 2019 Sody, Uddin, Grüneboom, Görgens, Giebel, Gunzer and Brandau. This is an open-access article distributed under the terms of the Creative Commons Attribution License (CC BY). The use, distribution or reproduction in other forums is permitted, provided the original author(s) and the copyright owner(s) are credited and that the original publication in this journal is cited, in accordance with accepted academic practice. No use, distribution or reproduction is permitted which does not comply with these terms. 